\title{
An anatomical study on locations of the mandibular foramen and the accessory mandibular foramen in the mandible and their clinical implication in a Thai population
}

\author{
Siriwat Thunyacharoen ${ }^{1}$, Solos Lymkhanakhom ${ }^{1}$, Pranpriya Chantakhat ${ }^{1}$, Supakorn Suwanin ${ }^{1}$,
} Supanat Sawanprom ${ }^{1}$, Anak Iamaroon ${ }^{2}$, Apirum Janhom², Pasuk Mahakkanukrauh ${ }^{3,4}$

${ }^{1}$ Medical Student, Faculty of Medicine, Chiang Mai University, Chiang Mai, ${ }^{2}$ Department of Oral Biology and Diagnostic Sciences, Faculty of Dentistry, Chiang Mai University, Chiang Mai, ${ }^{3}$ Department of Anatomy, Faculty of Medicine, Chiang Mai University, Chiang Mai, ${ }^{4}$ Excellence in Osteology Research and Training Center (ORTC), Chiang Mai University, Chiang Mai, Thailand

\begin{abstract}
Mandibular foramen (MF) is a structure that inferior alveolar nerve and artery pass through itself which is found on the mandible. The objective of this research aims to locate MF among the Thai population including other MF characteristics. The sampling is conducted in the Thai population of the total number of 220 samples from the Forensic Osteology Research Center, Faculty of Medicine, Chiang Mai University. The MF anteroposterior and superoinferior localizations are similar in both sex which at $\mathrm{Q}_{3}$ of right anteroposterior, $\mathrm{Q}_{2}$ of left anteroposterior and $\mathrm{Q}_{2}$ of left and right superoinferior axis. Otherwise, the prevalence of accessory MF has in 68 samples which are $30.89 \%$. The prevalence of unilateral single accessory MF is $20.45 \%$, bilateral single accessory MF is $6.36 \%$, unilateral double accessory MF is $2.27 \%$ and bilateral double accessory MF is $0.45 \%$. This present research results that the Thai population has a difference in MF location while comparing to other populations. Moreover, Thai MF and accessory MF location and localization will be helpful to clinical implications.
\end{abstract}

Key words: Mandibular foramen, Inferior alveolar nerve block

Received April 3, 2020; 1st Revised April 25, 2020; 2nd Revised May 7, 2020; 3rd Revised May 21, 2020; Accepted May 24, 2020

\section{Introduction}

The mandibular foramen (MF) is a superior opening which is found in the medial surface of the ramus of the mandible [1]. It has an important role to be a passage of mandibular canal which contains neurovascular structures

\footnotetext{
Corresponding author:

Pasuk Mahakkanukrauh (iD

Department of Anatomy \& Excellence in Osteology Research and Training Center (ORTC), Chiang Mai University, Chiang Mai 50200, Thailand

E-mail: pasuko34@gmail.com
}

such as inferior alveolar nerve, which is a branch of posterior trunk of trigeminal nerve that is sensory to the mucosa and skin around the lower lip and chin, and inferior alveolar artery, which is a branch of maxillary artery that goes through mental foramen [1].

MF has an important implication in dental operations, especially in the inferior alveolar nerve block [1]. So, anesthetic or surgical procedures are required a specific location of the MF, to perform precisely and appropriately, to prevent the operation failure which found mostly because of anatomical variation [2]. One anatomical variation can be found is accessory MF which is a foramen that usually reported as an unnamed foramen in the body of ramus of the mandible [3]. 
The inferior alveolar nerve and artery are also reported to also be seen in this accessory foramen [4]. There are studies of the prevalence of inferior alveolar nerve block failure is related to the presence of accessory foramen $[4,5]$.

Therefore, the anatomical study of the location of MF and prevalence of accessory MF is important for the inferior alveolar nerve block to prevent its failure, especially among the Thai population [5] because there are no collections of data in Thailand yet. So, knowledge of the location of MF and the prevalence of accessory MF in the Thai population in this study will be helpful for other anatomical and dental studies $[2,6]$.

This study aims to study the location of MF from various anatomical landmarks and prevalence of accessory MF from dry mandibles of the Thai population. The study of localization in anteroposterior and superoinferior axes are also noted.

\section{Materials and Methods}

\section{Samples}

This study conducted a cross-sectional descriptive study to examine mandibular bones from the bone bank of the Forensic Osteology Research Center, Anatomy Department, Chiang Mai University. The male samples were 110 bones and the female samples were 110 bones. Collected mandibular samples needed to be from adults (more than 20 years old cadavers) and have at least two teeth in sockets to determine occlusion plane of samples. Exclusively, the damaged bones or bone with pathological diseases such as congenital anomalies, osteoporosis will be uncollected in this study.

\section{Measurement}

To locate MF, various parameters were measured by digital Vernier calipers of $0.02 \mathrm{~mm}$ accuracy on both sides of the mandibles as in Fig. 1:

1. MF-AB: distance from the midpoint of the MF to the anterior border $(\mathrm{AB})$ of the ramus on occlusion plane

2. MF-PB: distance from the midpoint of the MF to the posterior border $(\mathrm{PB})$ of the ramus on occlusion plane

3. AB-PB: distance from the $A B$ of the ramus on occlusion plane to the $\mathrm{PB}$ of ramus passing midpoint of the $\mathrm{MF}$ or the summation of MF-AB and MF-PB

4. MF-MN: distance from the midpoint of the MF to the lowest point of the mandibular notch $(\mathrm{MN})$

5. MF-IB: distance from the midpoint of the MF to the inferior border (IB) limited to the base of the mandible

6. MN-IB: distance from the lowest point of the MN to the IB limited to the base of mandible passing midpoint of the MF or the summation of MF-MN and MF-IB

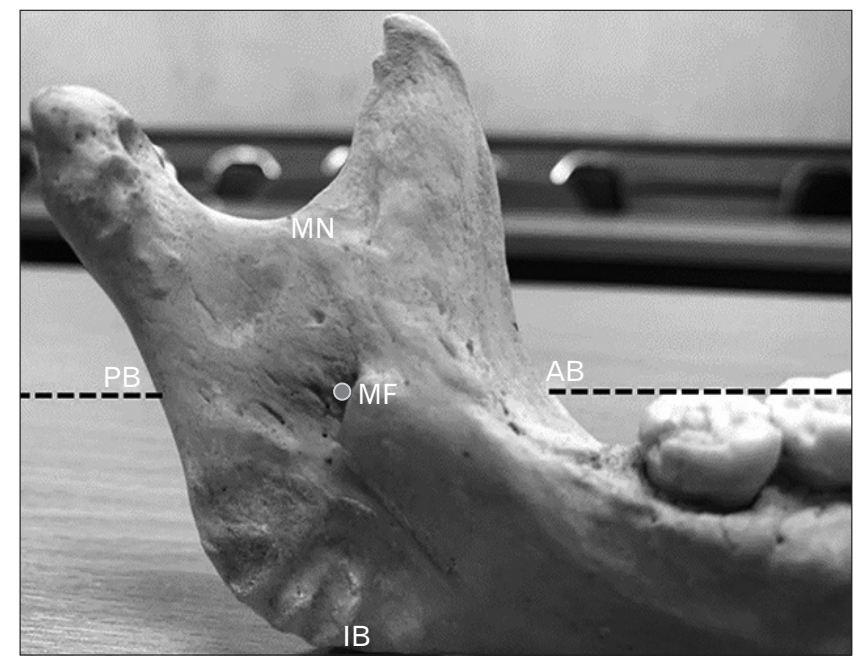

Fig. 1. Parameters measurements from various anatomical landmarks to MF. 1. MF-AB: distance from the midpoint of the MF to the AB of the ramus on occlusion plane. 2. MF-PB: distance from the midpoint of the MF to the PB of the ramus on occlusion plane. 3. AB-PB: distance from the $\mathrm{AB}$ of the ramus on occlusion plane to the $\mathrm{PB}$ of ramus passing midpoint of the MF or the summation of MF-AB and MF-PB. 4. MF-MN: distance from the midpoint of the MF to the lowest point of the MN. 5. MF-IB: distance from the midpoint of the MF to the IB limited to the base of the mandible. 6. MN-IB: distance from the lowest point of the MN to the IB limited to the base of mandible passing midpoint of the MF or the summation of MF-MN and MF-IB. AB, anterior border; IB, inferior border; MF, mandibular foramen; MN, mandibular notch; PB, posterior border; ---, occlusion plane; $\bullet$, midpoint of the MF. 
To be more precise, the location of MF was identified by the calculation of the prior parameters. Anteroposterior localization was calculated by percentile relation of $\mathrm{MF}-\mathrm{AB}$ to the AB-PB and superoinferior localization was calculated by percentile relation of MF-MN to the MN-IB. The quartiles of MF both anteroposteriorly and superoinferiorly were indicated by the value from $0 \%$ to $25 \%$, the first quartile; from $26 \%$ to $50 \%$, the second; from $51 \%$ to $75 \%$, the third and from $76 \%$ to $100 \%$, the fourth as in Fig. 2.

Further study, observation of the presence of accessory MF on both sides of mandibles by normal visual observation as Fig. 3.

The intra-observation was collected by the repetition of measurement similar parameter by one person three times from 20 samples, and inter-observation also was collected by another person measured parameter compared to mean of data from intra-observation from similar 20 samples. This process will give more reliability to the measurement procedure.

\section{Statistics analysis}

The various parameters indicated the location of MF was calculated to figure out mean and standard deviation. The parameters were compared between mandibular foramina in different sex. Also, the parameters were compared between both sides of mandibles among total samples, male samples and female samples.

The percentile of anteroposterior and superoinferior localization were calculated mean and standard deviation to find quartiles of MF in each mandible. The modest quartile of foramen would obtain a representative quartile of localization.

The accessory MF observations were calculated frequency

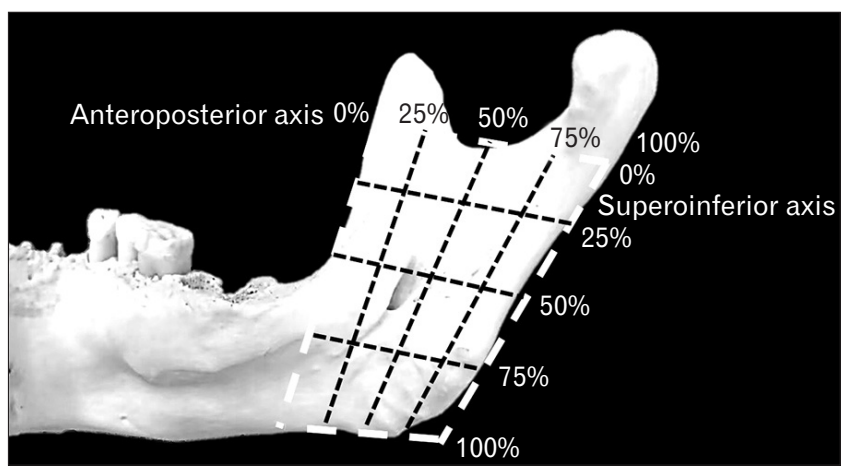

Fig. 2. Mandibular foramen localization in anteroposterior and superoinferior axes. and percentage classified by the absence of accessory MF, single accessory MF either right or left side, double accessory foramina either right or left side, bilateral accessory foramen, and single accessory foramen on one side and double accessory foramina on another side.

All the prior parameters were carefully statistic analyzed by IBM SPSS Statistics for Windows, Version 26.0 (IBM Co., Armonk, NY, USA) and Microsoft Excel 2016 (Microsoft Corp., Redmond, WA, USA). The descriptive analysis was employed for describing the central tendency and dispersion of data and an independent sample $t$-test was used as a test of significance under $P$-value $<0.05$ was considered as statistical significance.

\section{Results}

\section{The distance of mandibular foramen from various mandibular landmarks on the right and left sides among all samples}

Measurements of distance from various mandibular landmarks from 220 samples are studied and resulted in Table 1. The comparisons of mean and standard deviation of parameters between the right side and left side mostly have no statistically significant difference $(P>0.05)$ except for MF-AB and AB-PB parameters $(P<0.05)$.

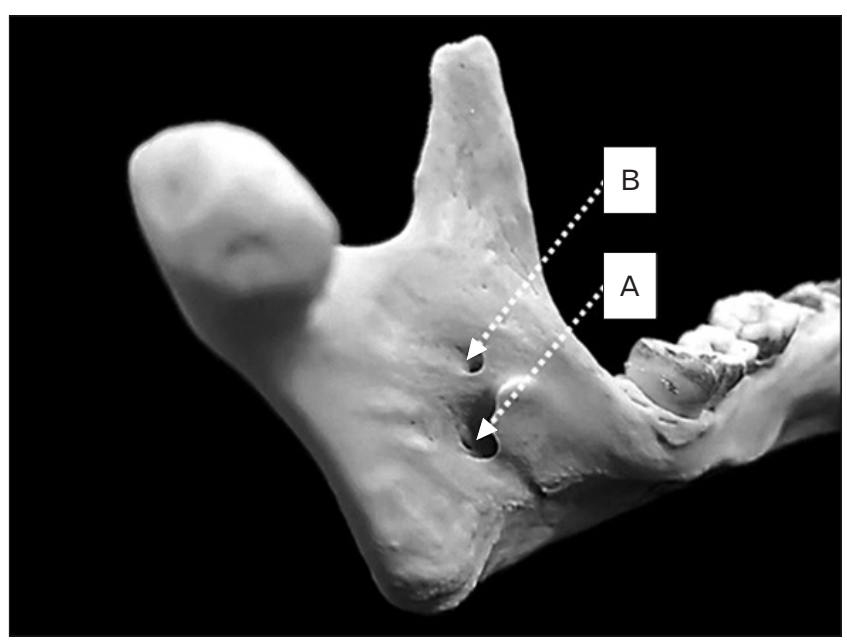

Fig. 3. Accessory mandibular foramen observation. A, mandibular foramen; $\mathrm{B}$, accessory mandibular foramen. 


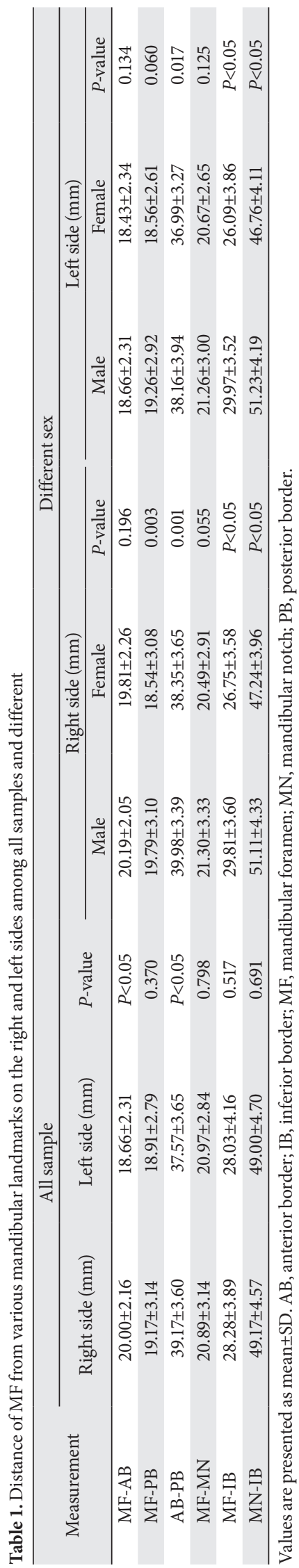

\section{The distance of mandibular foramen from various mandibular landmarks on the right and left sides in different sex}

Samples are classified by different sex, the mean and standard deviation of various parameters between male samples and female parameters were studied and resulted in Table 1 . The comparisons between different sex have no statistically significant difference in MF-AB, MF-PB and MF-MN parameters $(P>0.05)$. Meanwhile, between different sex AB-PB, MF-IB and MN-IB parameters have a statistically significant difference $(P<0.05)$ on both sides and MF-PB parameter has a statistically significant difference $(P<0.05)$ in left side.

\section{Localization of mandibular foramen in the anteroposterior and superoinferior axis of ramus of the mandible on the right and left sides among all samples}

Percentile of anteroposterior localization of MF on the right side is found averagely $51.25 \% \pm 5.20 \%$ at the third quartile, while on the left side is found $49.75 \% \pm 4.77 \%$ at the second quartile. Superoinferior axis localization on the right side is found in percentile $45.54 \% \pm 5.40 \%$ or at the second quartile, likely on the left side is found $42.92 \% \pm 5.27 \%$ at the second quartile. The results are shown in Table 2. According to the independent sample $t$-test, anteroposterior localization has a statistically significant difference $(P=0.002)$. In contrast, superoinferior localization has no statistically significant difference $(P=0.452)$.

\section{Localization of mandibular foramen in the anteroposterior and superoinferior axis of ramus of the mandible on the right and left sides among male samples}

Among male samples, right anteroposterior MF localization is averagely in percentile $50.67 \% \pm 5.09 \%$ at the third quartile and right superoinferior MF localization is averagely in $41.65 \% \pm 5.25 \%$ at the second quartile. Meanwhile, left anteroposterior MF localization is averagely $49.63 \% \pm 4.51 \%$ at the second quartile and left superoinferior MF is averagely in $41.50 \% \pm 4.82 \%$ at the second quartile. The independent sample $t$-test has resulted in no statistically significant differences both in the anteroposterior $(P=0.110)$ and superoinferior axis $(P=0.827)$. The analysis results are shown in Table 2 .

\section{Localization of mandibular foramen in the anteroposterior and superoinferior axis of ramus of the mandible on the right and left sides among female samples}

Among female samples, right anteroposterior MF local- 


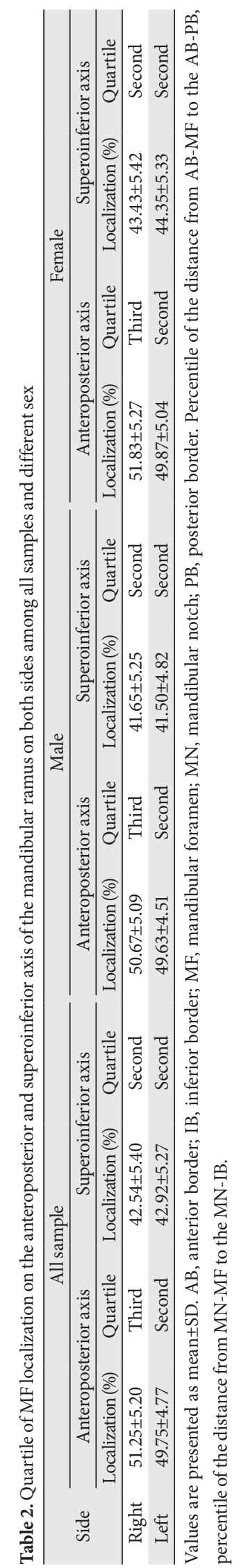

ization is averagely in percentile $51.83 \% \pm 5.27 \%$ at the third quartile and right superoinferior MF localization is averagely in $43.43 \% \pm 5.42 \%$ at the second quartile. Meanwhile, left anteroposterior MF localization is averagely $49.87 \% \pm 5.04 \%$ at the second quartile and left superoinferior MF is averagely in $44.35 \% \pm 5.33 \%$ at the second quartile. The independent sample $t$-test has resulted in the anteroposterior axis has a statistically significant difference $(P=0.05)$, yet the superoinferior axis has no statistically significant difference $(P=0.208)$. The analysis results are shown in Table 2 .

\section{Prevalence of the accessory mandibular foramen}

The observations of accessory mandibles are found that 68 samples (from total 220 samples) have accessory MF which is $30.9 \%$. Distinguishly, the unilateral single accessory MF is found 45 samples, which calculate be $20.5 \%$, the bilateral single accessory MF is found 14 samples, which calculate be $6.4 \%$, the unilateral double accessory MF is found 5 samples, which calculate be $2.3 \%$, the bilateral accessory MF is found 1 sample, which calculates be $0.5 \%$, the right single accessory MF with left double accessory MF is found 2 samples, which calculate be $0.9 \%$, and the left single accessory MF with right double accessory MF is found 1 sample, which calculates be $0.5 \%$. The numbers and percentages of the prevalence of accessory MF are Table 3.

\section{Intra-observational and inter-observational measurement}

\section{Intra-observational measurement}

Four parameter observations found the F-value and $P$ value showed no statistically significant differences mean of measurements at least 1 time in each sample measurement $(\mathrm{F}<3.158, P>0.05)$. In another way, there is no statistically significant difference of mean in any pairs of a similar sample. The intra-observation was calculated reliability by using Fvalue and $P$-value of one-way ANOVA test that reflect selfreliability of measurement.

\section{Inter-observational error measurement}

The inter-observer error is performed on 4 parameters to evaluate their reliability and repeatability. Twenty cases randomly were selected and remeasured by 2 observers. All measurement were compared by the technical error measurement (TEM) and coefficient of reliability (R) for estimating the precision. 
Table 3. Prevalence of accessory MF in 220 dry adult human mandibles

\begin{tabular}{lc}
\hline \multicolumn{1}{c}{ Accessory MF } & Value $(\mathrm{n}=220)$ \\
\hline Right side- single accessory foramen & $22(10.0)$ \\
Left side- single accessory foramen & $23(10.5)$ \\
Right side- double accessory foramina & $4(1.8)$ \\
Left side- double accessory foramina & $1(0.5)$ \\
Bilateral single accessory foramen & $14(6.4)$ \\
Bilateral double accessory foramina & $1(0.5)$ \\
Right side- single accessory foramen with & $2(0.9)$ \\
left side- double accessory foramina & $1(0.5)$ \\
Left side- single accessory foramen with & \\
right side- double accessory foramina & $152(69.1)$ \\
Absent accessory foramen & $220(100.0)$ \\
\hline All
\end{tabular}

Values are presented as number (\%). MF, mandibular foramen.

The inter-observer error statistics for 4 parameters have shown in the following. AB-MF measurement has TEM=0.694 $\mathrm{mm}, \mathrm{R}=0.906$; PB-MF measurement has $\mathrm{TEM}=0.585 \mathrm{~mm}$, $\mathrm{R}=0.776$; $\mathrm{MN}-\mathrm{MF}$ measurement has $\mathrm{TEM}=0.636 \mathrm{~mm}$, $\mathrm{R}=0.986$ and $\mathrm{IF}-\mathrm{MF}$ measurement has $\mathrm{TEM}=0.432 \mathrm{~mm}$, $\mathrm{R}=0.989$. TEM or $\mathrm{R}$ statistics flag the error well.

\section{Discussion}

This study of the location of MF in the Thai population has difference MF location from other international studies in means, standard deviations and statistically significant differences between the left and right sides of mandibles. Sella Tunis et al. studied that anatomical structure of mandible is significantly correlated to masticatory muscles crosssectional area, which are temporalis muscle and masseter muscle [7]. In other words, if muscle mass increases, the mandible structure will be e.g. widening ramus, larger coronoid of the mandible. Increasing muscle mass can result in some pathologic conditions such as TMJ disorders, psychological disorders or physiological conditions such as chewing [8]. Simione et al. [9] reported structural differences in foods especially the hardness of food is affecting chewing performance. Therefore, a variety of food which is mostly related to masseter muscle increasing in size, affecting the mandible structure, leading to different MF location in different ethnicity as Table $4[5,10,11,12]$.

Ennes and Mediros [10] studied MF location in Brazil population found the means and standard variations from various anatomical landmarks: Right $M F-A B$ is $9.40 \pm 2.03 \mathrm{~mm}$, left MF-AB is $6.90 \pm 2.06 \mathrm{~mm}$, right $\mathrm{MF}-\mathrm{PB}$ is $8.60 \pm 1.20 \mathrm{~mm}$, left MF-PB is $8.40 \pm 1.77 \mathrm{~mm}$, right MF-MN is
$18.30 \pm 3.25 \mathrm{~mm}$, left MF-MN is $17.50 \pm 3.37 \mathrm{~mm}$, and means from left and right sides have no statistically significant difference. Ennes and Mediros [10] studied MF localization in Brazil population found the right and left anteroposterior localizations are at $56.43 \%$ and $56.33 \%$ respectively. They are in the third quartile similarly. Moreover, right and left superoinferior localization are at $53.27 \%$ and $52.43 \%$ respectively which are also at the third quartile [9]. In addition, Oguz and Bozkir [11] have investigated to localize the MF in adult dry bone of Turkish population. Right MF-AB is $16.9 \mathrm{~mm}$, left $\mathrm{MF}-\mathrm{AB}$ is $16.78 \mathrm{~mm}$, right MF-PB is $14.09 \mathrm{~mm}$, left $\mathrm{MF}-\mathrm{PB}$ is $14.37 \mathrm{~mm}$, right MF-MN is $22.37 \mathrm{~mm}$, left MF-MN is 22.17 $\mathrm{mm}$ with no SD value.

Padmavathi et al. [12] reported MF localization from the South Indian population found right and left anteroposterior localizations are at the third quartile. Differently, right and left superoinferior localization are at the junction between the second and third quartile.

Shalini et al. [5] studied MF location in South Indian population found the means and standard variations from various anatomical landmarks: Right MF-AB is $17.11 \pm 2.74$ $\mathrm{mm}$, left MF-AB is $17.41 \pm 3.05 \mathrm{~mm}$, right $\mathrm{MF}-\mathrm{PB}$ is $10.47 \pm 2.11 \mathrm{~mm}$, left MF-PB is $9.68 \pm 2.03 \mathrm{~mm}$, right MF-MN is $21.74 \pm 2.74 \mathrm{~mm}$, left MF-MN is $21.92 \pm 3.33 \mathrm{~mm}$, and means from left and right sides have no statistically significant difference. This present study has differences from previous studies which are Right MF-AB is $20.00 \pm 2.16 \mathrm{~mm}$, left MF$\mathrm{AB}$ is $18.66 \pm 2.31 \mathrm{~mm}$, right $\mathrm{MF}-\mathrm{PB}$ is $19.17 \pm 3.14 \mathrm{~mm}$, left MF-PB is $18.91 \pm 2.79 \mathrm{~mm}$, right MF-MN is $20.89 \pm 3.14 \mathrm{~mm}$, left MF-MN is $20.97 \pm 2.84 \mathrm{~mm}$ and there are some statistically significant differences between left and right sides in AB-PB, MF-IB, MN-IB parameters which is differed from previous studies showed no statistically significant difference in these parameters. Nonetheless, between male and female AB-PB, MF-IB and MN-IB parameters also have a statistically significant difference. Besides of location of the MF, the localization of MF is also noted finding the difference between this study and previous studies as Table $4[5,10,11,12]$. Shalini et al. [5] also reported MF localization from South Indians found right and left anteroposterior localizations are at $56.73 \% \pm 3.44 \%$ and $62.2 \% \pm 2.32 \%$ respectively. They are in the third quartile similarly. Moreover, right and left superoinferior localization are at $49.68 \% \pm 3.46 \%$ and $46.51 \% \pm 5.10 \%$ respectively which are at the junction between the second and third quartile. This present study has differences from previous studies that are right and left anteroposterior local- 


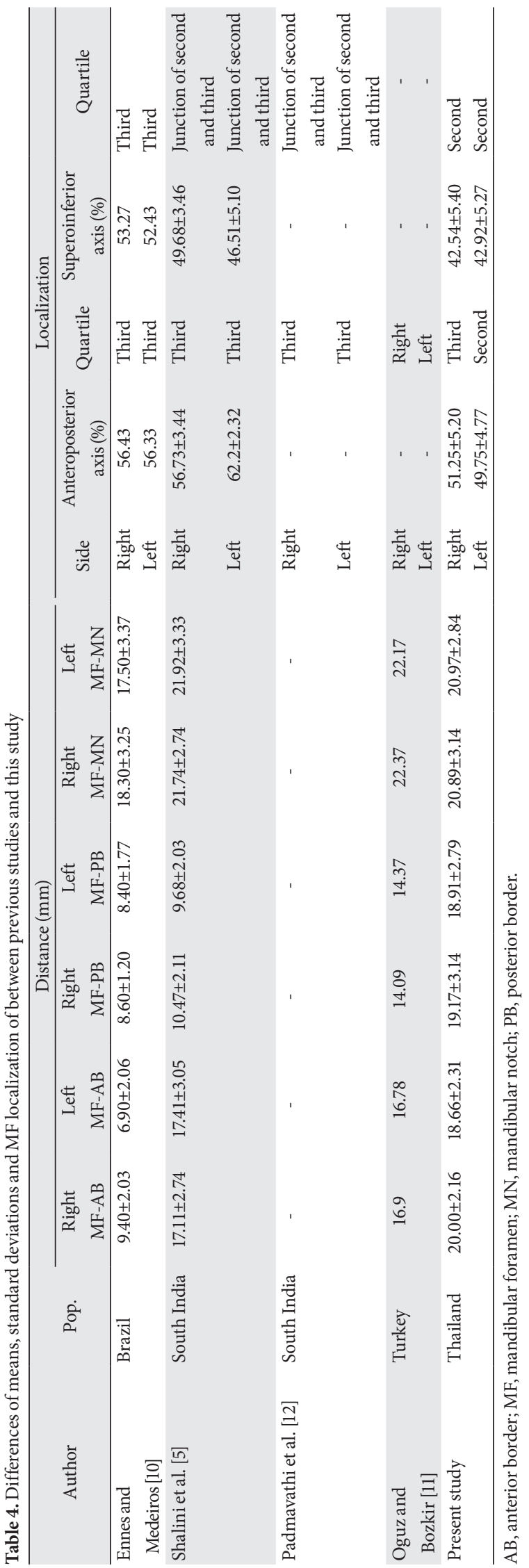

izations are at $51.25 \% \pm 5.20 \%$ and $49.75 \% \pm 4.77 \%$ which are at the third and second quartile respectively. Moreover, right and left superoinferior localization are at $42.54 \% \pm 5.40 \%$ and $42.92 \% \pm 5.27 \%$ respectively which are at the third quartile. Hence, there is a statistically significant difference in anteroposterior localization between the left and right sides. On another side, there is no statistically significant difference in localizations between males and females.

Accessory MF that contains accessory inferior alveolar nerve is due to embryogenesis, initially, the inferior alveolar nerve has 3 branches. Later, the branches of the nerve will be fused together as one inferior alveolar nerve but in a variation of the accessory foramen, the branches can be separated and passed through the normal MF and accessory MF [12]. The studies of the prevalence of accessory MF are shown in Table $5[5,13,14]$.

Shalini et al. [5] found out that the prevalence of accessory MF in the South Indian population has unilateral foramen $22.05 \%$, bilateral foramen $10.30 \%$, so, in total $32.36 \%$. While Galdames et al. [13] found prevalence in Brazil differently which is unilateral foramen $23.40 \%$, bilateral foramen $19.10 \%$, in total $42.60 \%$. Additionally, Lima et al. [14] also found prevalence in Brazil population which is unilateral foramen $26.60 \%$, bilateral foramen $13.30 \%$, in total $50.00 \%$. This study yet conducted prevalence of accessory MF classified as unilateral foramen $22.72 \%$, bilateral foramen $6.81 \%$, right single accessory MF with left doubled accessory MF $0.91 \%$, left single accessory MF and right doubled accessory MF $0.45 \%$. which concluding in total $30.89 \%$

The implication of location and localization of MF in the Thai population can be done in many dental surgical and anesthetic procedures [14]. The location of MF may be reached from anatomical landmark especially IB of the ramus of mandibles due to physical examination of jawline before the procedure. The statistical analysis showed the statistical significance of the MF-IB parameter between males and females. So, there can be some estimations of the location of mandible foramen differently in males and females before any procedure. As well as localization, there is a statistically significant difference between the right and left side of anteroposterior localization. The right anteroposterior localization is at the third quartile, while left anteroposterior localization is at the second quartile. Accordingly, the alveolar nerve block depth of injection should be done differently which right side may be deeper than the left side. The prevalence of accessory MF is still important to the inferior 
Table 5. Differences of the prevalence of accessory MF between previous studies and this study

\begin{tabular}{llcccc}
\hline \multicolumn{1}{c}{ Author } & Population & Unilateral (\%) & Bilateral (\%) & $\begin{array}{c}\text { One-sided unilateral-one } \\
\text { sided bilateral (\%) }\end{array}$ & Total (\%) \\
\hline Shalini et al. [5] & South India & 22.05 & 10.30 & - & 32.36 \\
Galdames et al. [13] & Brazil & 23.40 & 19.10 & - & 42.60 \\
Lima et al. [14] & Brazil & 26.60 & 23.30 & - & 50.00 \\
Present study & Thailand & 22.72 & 6.81 & 1.36 & 30.89 \\
\hline
\end{tabular}

MF, mandibular foramen.

alveolar nerve block. Even though the location of the accessory MF is not significantly affecting the injection but the presence of accessory MF may affect the dosage of anesthetic injection which infers as the presence of accessory MF may need more doses of anesthetic injection. Among Thai population found around one-third of the population has accessory MF who may need a higher dosage of anesthetic injection in inferior alveolar nerve block.

In this study, the measurement of parameters is also finding the reliability of measurement by compare intra-observer error and inter-observer error. The intra-observer error is tested the hypothesis by one-way ANOVA test finding there is no statistically significant difference within each observer (accepting $\mathrm{H}_{0}$ hypothesis).

In conclusion, according to the study of location and localization of MF and prevalence of accessory MF in Thai population found differences between ethnicities. This knowledge can be applied in clinically dental surgical and anesthetic procedures especially inferior alveolar nerve block.

\section{ORCID}

Siriwat Thunyacharoen:

https://orcid.org/0000-0002-8654-349X

Solos Lymkhanakhom:

https://orcid.org/0000-0003-2094-145X

Pranpriya Chantakhat:

https://orcid.org/0000-0002-7034-3194

Supakorn Suwanin:

https://orcid.org/0000-0002-3630-620X

Supanat Sawanprom:

https://orcid.org/0000-0002-2375-9293

Anak Iamaroon: https://orcid.org/0000-0002-8703-5083

Apirum Janhom: https://orcid.org/0000-0002-8485-5684

Pasuk Mahakkanukrauh

https://orcid.org/0000-0003-0611-7552

\section{Author Contributions}

Conceptualization: ST, PM. Data acquisition: ST, SL, PC, SS, SS. Data analysis or interpretation: ST, SL, PC, SS, SS. Drafting of the manuscript: ST, SL, PC, SS, SS. Critical revision of the manuscript: AI, AJ, PM. Approval of the final version of the manuscript: all authors.

\section{Conflicts of Interest}

No potential conflict of interest relevant to this article was reported.

\section{Acknowledgements}

This study was partially supported by the research administration section from the Faculty of Medicine (Grant number: 093/2563) and the Excellence in Osteology Research and Training Center (ORTC), Chiang Mai University. The author would like to thank to those who donated the body for study and research.

\section{References}

1. Drake RL, Vogl AW, Mitchell AWM. Gray's anatomy for students. 2nd ed. Philadelphia: Elsevier; 2010.

2. Khoury J, Townsend G. Neural blockade anaesthesia of the mandibular nerve and its terminal branches: rationale for different anaesthetic techniques including their advantages and disadvantages. Anesthesiol Res Pract 2011;2011:307423.

3. Rao TR, Chitturi RT, Sudhiksha S, Rao SR. Presence of accessory mandibular foramina and their clinical implications. Case Stud J 2017;6:14-7.

4. Gupta S, Soni A, Singh P. Morphological study of accessory foramina in mandible and its clinical implication. Indian J Oral Sci 2013;4:12.

5. Shalini R, RaviVarman C, Manoranjitham R, Veeramuthu M. Morphometric study on mandibular foramen and incidence of accessory mandibular foramen in mandibles of south Indian population and its clinical implications in inferior alveolar 
nerve block. Anat Cell Biol 2016;49:241-8.

6. Khalil H. A basic review on the inferior alveolar nerve block techniques. Anesth Essays Res 2014;8:3-8.

7. Sella-Tunis T, Pokhojaev A, Sarig R, O'Higgins P, May H. Human mandibular shape is associated with masticatory muscle force. Sci Rep 2018;8:6042.

8. Rispoli DZ, Camargo PM, Pires JL Jr, Fonseca VR, Mandelli KK, Pereira MAC. Benign masseter muscle hypertrophy. Braz J Otorhinolaryngol 2008;74:790-3.

9. Simione M, Loret C, Le Révérend B, Richburg B, Del Valle M, Adler M, Moser M, Green JR. Differing structural properties of foods affect the development of mandibular control and muscle coordination in infants and young children. Physiol Behav 2018;186:62-72.

10. Ennes JP, de Medeiros RM. Localization of mandibular fora- men and clinical implications. Int J Morphol 2009;27:1305-11.

11. Oguz O, Bozkir MG. Evaluation of location of mandibular and mental foramina in dry, young, adult human male, dentulous mandibles. West Indian Med J 2002;51:14-6.

12. Padmavathi G, Tiwari S, Varalakshmi KL, Roopashree R. An anatomical study of mandibular and accessory mandibular foramen in dry adult human mandibles of South Indian origin. IOSR J Dent Med Sci 2014;13:83-8.

13. Galdames ICS, Matamala DAZ, Smith RL. Is the conduct of Serres an anatomical variation in adults? Int J Morphol 2009;27:43-7.

14. Lima FJC, Oliveira Neto OB, Barbosa FT, Dantas LCS, Olave E, Sousa-Rodrigues CF. Occurrence of the accessory foramina of the mandibular ramus in Brazilian adults and its relation to important mandibular landmarks. Int J Morphol 2016;34:330-4. 